PACS 75.50.Dd

\title{
Numerical simulation of the generation of multisoliton type magnetic inhomogeneities in ferromagnets with inhomogeneous parameters
}

\author{
E. G. Ekomasov, A. M. Gumerov ${ }^{\dagger}$ \\ †bgu@bk.ru
}

Bashkir State University, 32 Z. Validi St., 450076, Ufa, Russia

\begin{abstract}
The generation and evolution of multisoliton type magnetic inhomogeneities, which appear in two flat layers with the magnetic anisotropy that are different from those in three thick layers after pinning a $180^{\circ}$ domain wall, have been investigated theoretically. The structure of the multisoliton type magnetic inhomogeneities have been constructed for the revealed magnetic inhomogeneities, and the ranges of the parameters determining the possibility of their existence have been found.
\end{abstract}

Keywords: three-layer ferromagnet, domain wall dynamics, modified sine-Gordon equation.

\section{Introduction}

Different types of structural and chemical inhomogeneities involved in real magnets, as well as local (mechanical, thermal, or light) effects, give rise to local variations in magnetic parameters of the material. The existence of these inhomogeneities (or defects) in magnetic materials can lead to the appearance of spatially localized vibrational modes and the formation of different types of magnetic inhomogeneities, which affect the magnetization reversal of the sample [1]. Since it is usually difficult to perform the exact (microscopic) calculation, it is necessary to simulate the functions describing the parameters of the inhomogeneous material (for example, magnetic anisotropy constant, exchange interaction parameter, etc.). For ferromagnetic materials, it is common practice to use the approximation of the defect in the form of a planar (or plate like) magnetic inclusion, which is considered to be finite in thickness $[2,3]$. The influence of planar magnetic inclusions on the static and some dynamic properties of magnetic inhomogeneities was investigated using both the analytical and numerical methods [2-7]. It should be noted that multilayer magnetic structures are investigated using the models that take into account both the local and periodic spatial modulations of the magnetic parameters of the material (see, for example, $[8,9]$ ).

At the same time, the inclusion of the spatial dependence of the material parameters in the analysis of the domain wall (DW) dynamics leads to a mathematically interesting problem of finding the solution to the modified sine-Gordon equation with variable coefficients, which is of great importance for many fields of the modern physics [10]. It is also known that, in these systems, apart from conventional linear excitations, namely, spin waves, there are nonlinear excitations, such as, for example, breathers [11]. The magnetic inhomogeneities generated on defects are frequently described as the $0^{\circ}$ domain walls (or magnetic soliton) $[3,12]$. It should be noted that, in a defect-free magnet, magnetic inhomogeneities of $0^{\circ}$ domain wall type are energetically unfavorable as compared to the homogeneous state of the magnetization vector. The presence of the defects that locally change the sign of the uniaxial anisotropy in the crystal can lead to the energetic favorability of $0^{\circ}$ domain walls, as well as to their generation in the presence of excitations in the system [11]. In this work, we have considered the excitation conditions of localized high-amplitude nonlinear waves in the region of two planar magnetic inclusions.

\section{Basic equations and results exciting multisolitons on impurities}

Let us consider an infinite ferromagnet in which the crystallographic axes $(a, b, c)$ coincide with the Cartesian coordinate axes $(x, y, z)$. By considering that the energy density of the magnet takes into account the exchange interaction and anisotropy, the equation of motion for the magnetization in the angular variables $\mathbf{m}=\mathbf{m}(0, \sin \theta, \cos \theta)$ can be represented in the dimensionless form [11]

$$
\frac{\partial^{2} \theta}{\partial t^{2}}-\frac{\partial^{2} \theta}{\partial x^{2}}+\frac{K(x)}{2} \sin 2 \theta=0
$$

where $\mathbf{m}$ is the ferromagnetic vector, $\theta$ is the angle in the $y z$ plane between the direction of the vector of the magnetic moment $\mathbf{m}$ and the easy magnetization axis (the $\mathrm{O} z$ axis). $K(x)$ is the function that determines the distribution of the inhomogeneity of the anisotropy constant. The coordinate $x$ is normalized to $\delta_{0}$ (where $\delta_{0}$ is the width of the static Bloch domain wall), and the time $t$ is normalized to $\delta_{0} / c$ (where 
$c$ is the Walker limiting velocity of the steady state motion [1]). In case $K(x)=1$ the Eq.(1) goes over into the known sine-Gordon equation and is solved as a topological soliton or kink:

$$
\theta(x, t)=2 \arctan \left(\exp \left[\Delta\left(v_{0}\right)\left(x-v_{0} t\right)\right]\right.
$$

where $\Delta(v)=\left(1-v^{2}\right)^{-1 / 2}, v_{0}$ is an continuous parameter $\left(0<v_{0}<1\right)$ determining the DW velocity. Also, there exists a spat ial localized solution of Eq.(1) as a stationary breather:

$$
\theta_{\text {breather }}(x, t, \omega)=2 \arctan \left(\frac{\sqrt{1-\omega^{2}} \sin \omega t}{\omega \cosh \left(\sqrt{1-\omega^{2}}\left(x-x_{0}\right)\right)}\right)
$$

where $\omega$ is the breather oscillation frequency and $x_{0}$ its centre coordinate.

There are multisoliton solutions of the sine-Gordon equation. For instance, in $[13,14]$ an interesting threekink solution of a wobble type is described:

where

$$
f_{\text {wobble }}(x, t)=\frac{4}{\beta} \arctan \frac{A}{B}
$$

$$
\begin{aligned}
& A=\frac{\sqrt{1-\omega^{2}}}{\omega} \sin (m \omega t)+\frac{e^{\varepsilon m\left(x-x_{0}\right)}}{2}\left(e^{-m \sqrt{1-\omega^{2}}\left(x-x_{0}\right)}+\rho^{2} e^{m \sqrt{1-\omega^{2}}\left(x-x_{0}\right)}\right), \\
& B=\cosh \left(m \sqrt{1-\omega^{2}}\left(x-x_{0}\right)\right)+\frac{\sqrt{1-\omega^{2}}}{\omega} \rho e^{\varepsilon m\left(x-x_{0}\right)} \sin (m \omega t), \\
& \rho=\frac{1-\varepsilon \sqrt{1-\omega^{2}}}{1+\varepsilon \sqrt{1-\omega^{2}}},-1<\omega<1, \varepsilon= \pm 1
\end{aligned}
$$

$x_{0}$ is "the centre» coordinate of the solution (however, contrary to the kink (2) this parameter does not coincide with the geometry kink centre), $\omega$ is a wobble oscillation frequency. It is worth noting that $\beta, m, \varepsilon$ parameters admit to change the general form of the solution. Equation (1), which has been intensively studied recently, is a modified sineGordon equation with variable coefficients.

The most interesting case is the one where the size of the domain wall and the size characterizing the inhomogeneity of the parameters (or impurities) are of the same magnitude order; then, the shape of the domain wall must undergo substantial changes when it passes through the inhomogeneous region. The spatial modulation of the periodic potential is to be modelled as [2]:

$$
K(x)=\left\{\begin{array}{l}
1, x<x_{1}, x_{1}+W<x<x_{1}+W+d, x>x_{1}+2 W+d \\
1-\Delta K, x_{1} \leq x \leq x_{1}+W, x_{1}+W+d \leq x \leq x_{1}+2 W+d
\end{array}\right.
$$

i.e. as two identical impurities separated by a certain distance $d$. Obviously, at $\Delta K>0$, the impurity is a potential well for the moving kink whereas at $\Delta K<0$ it is a potential barrier.

The Eq.(1) was solved numerically using an explicit scheme. The equation discretization was carried out by the standard five-point scheme of the "cross" type with the stability condition $(\Delta t / \Delta h)^{2} \leq 0.5$, where $\Delta t$ is a time step and $\Delta h$ is a coordinate step. Initially we have the DW (2) moving at a constant velocity $v_{0}$, with boundary conditions of $\theta(-\infty, t)=0, \theta(+\infty, t)=\pi, \theta^{\prime}( \pm \infty, t)=0$. Common numerical realizations of the Eq.(1) used in $[11,15]$ enable us to calculate the kink structure and dynamics accurately enough to watch the kink pinning and passing through the impurities, structure and properties of the non-linear waves excited. Yet much higher accuracy is required to study possible resonance effects. So $N_{\mathrm{x}}=10^{4}$ points were used for approximating the function $\theta(x, t)$. Special control of the result errors was carried out.

We examine the case of the DW pinning in one of the impurities. Note that the presence of two impurity areas enables us to find multisoliton solutions of the sine-Gordon equation. To be definite enough, we take $W=1, \Delta K=1.2$, $x_{1}=-7$ and the distance $d$ between the impurities may vary widely. In all cases hereinafter the initial DW velocity was selected so that the kink was pinned in the second impurity area. There are some difficulties in the numerical study of the task considered. As the interaction of the solitons excited may lead to oscillation mode appearance characterized by energy transfer from the DW to the magnetic breather and conversely (similar to the beat regime for harmonic oscillators), the oscillation frequency may change in the course of time. So further, we resort to isotropic oscillations and stationary frequencies that are set over time.

At the beginning we consider the case when the parameter $d$ is high. As seen from Fig. 1 a at $d=10$ the DW is pinned in the second impurity area and transmission $\omega_{\text {trans }}$ and pulsation $\omega_{\text {pulse }}$ modes are excited whereas in the first impurity area the breather with $\omega_{\text {breather }}$ frequency is excited. The characteristic frequencies amount to $\omega_{\text {trans }}=0.429, \omega_{\text {pulse }}=0.875, \omega_{\text {breather }}=0.92$ (Fig.2). If we compare the values of the excited pulsation and transmission kink modes and oscillation breather frequencies for a single impurity [11], we shall see that they practically coincide. Thus, the soliton interaction in the impurity area is not observed at high d parameters. The obtained multisoliton consisting of the weakly interacting DW and magnetic breather is somehow connected with the well-known threekink solution of non-linear differential triton equations.

Let us study the changes in the triton structure and properties at decreasing parameter $d$. As seen from Fig.1, we obtain a triton consisting of the non-interacting DW and magnetic breather at $d=5$ and above. Starting with $d=4$ (Fig.1b), the triton structure and properties change greatly. The DW and magnetic breather are strongly bound, and inner oscillation kink modes (pulsation and transmission) become equal to the breather oscillation frequency (Fig.2). The obtained magnetic multisoliton can be considered as a special triton solution of the sine-Gordon equation of the wobble type (4) at $\beta=2, m=1, \varepsilon=1$.

In the third, most narrow area the formation of a strongly bound DW and magnetic soliton state is possible at $d \approx$ $1.4-1.6$ (Fig.1c) which is a three-kink state with much lower oscillation frequency $\omega_{\text {pulse }} \approx \omega_{\text {trans }} \approx \omega_{\text {breather }} \approx 0.46$ (Fig.2). It corresponds to the results [11] forsoliton oscillation frequency. For the triton case periodic energy transfer between the magnetic soliton and the DW is quite typical. The case is that according to [11] the chosen parameters $W$ and $\Delta K$ are to lead to the magnetic breather formation. Collectiveinteraction effects of the impurities at a short distance result in the fact that a magnetic soliton is excitedinstead of the magnetic breather. Further decrease of the distance between the defects $d<1.2$ makes it impossible to analyze the triton structure by the used numerical method. 

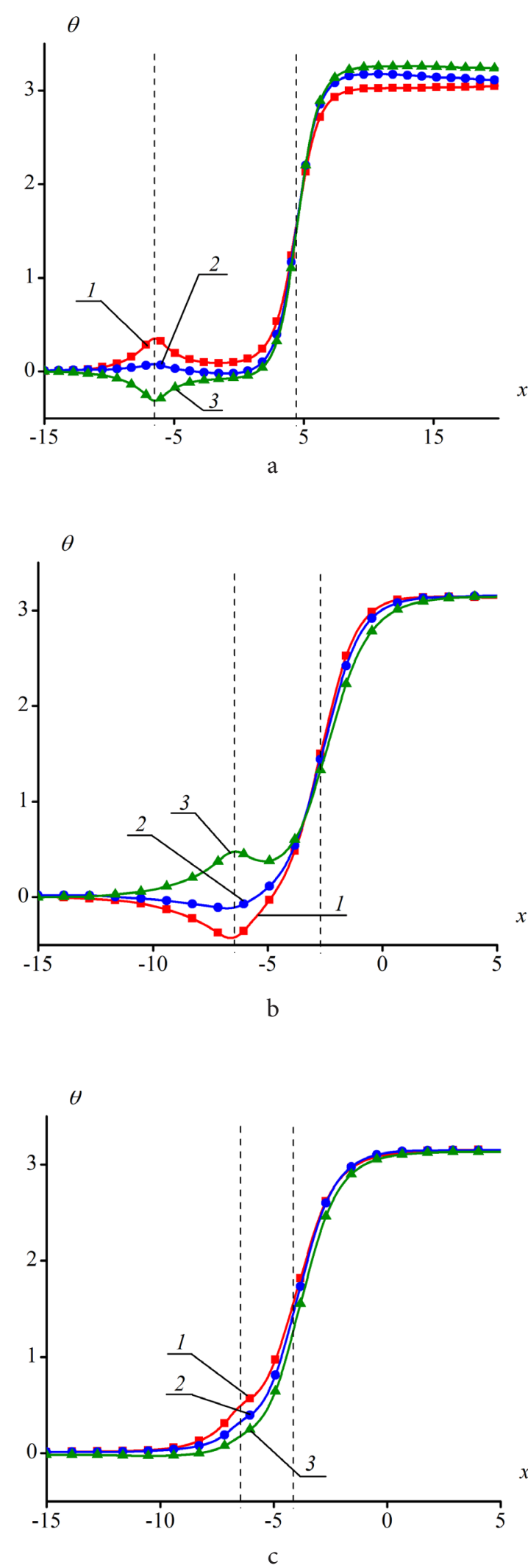

Fig. 1. The magnetic multisoliton profile $\theta(x)$ in different moments of time: (a) at $W=1, \Delta K=1 ; 2, d=10$ in $t_{1}=1308.41$, $t_{2}=1309.91, t_{3}=1311.45 ;$ (b) at $W=1, \Delta K=1 ; 2, d=3$ in $t_{1}=784.748, t_{2}=786.249, t_{3}=787.749 ;$ (c) at $W=1, \Delta K=1.2, d=$ 1.6 in $t_{1}=1549.99, t_{2}=1551.49, t_{3}=1554.49$. Dashed lines denote centre areas of the $1^{\text {st }}$ and $2^{\text {nd }}$ impurities.

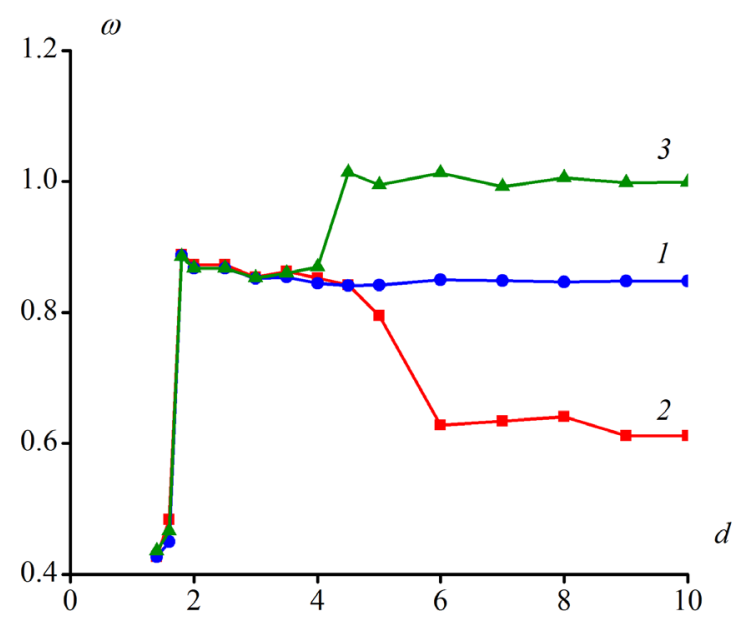

Fig. 2. Dependence of the magnetic breather frequency $\omega_{\text {breather }}$ (curve 1), pulsation DW mode $\omega_{\text {pulse }}$ (curve 2) and transmission DW modes $\omega_{\text {trans }}$ (curve 3 ) from the parameter $\mathrm{d}$ at $W=1, \Delta K=1.2$.

\section{Conclusion}

Pinning of the DW and exciting high amplitude localized nonlinear waves on the impurity may be used for multisoliton excitation in the sine-Gordon equation. A triton consisting of the weakly bound DW and magnetic breather is observed at long distances between the impurities. Starting with a certain critical distance pulsation and transmission mode frequencies are synchronized with the magnetic breather oscillation frequency and a triton solution of a wobble type is observed. At very short impurity distances excitation of the strongly bound DW and magnetic soliton is possible. The dependence of the structure and excited magnetic multisoliton frequencies from the impurity distances is determined.

\section{References}

1. A. Hubert and R. Schafer. Magnetic Domains, Springer, Heidelberg. (1998).

2. D. I. Paul. J. Phys. C: Solid State Phys. 12, 585 (1979).

3. M.A. Shamsutdinov, V.G. Veselago, M.M. Farztdinov, E. G. Ekomasov. Phys. Solid State. 32(2), 288 (1990).

4. E.G. Ekomasov, Sh.A. Azamatov, R.R. Murtazin, A. M. Gumerov, A. D. Davletshina. Bull. Russ. Acad. Sci.: Phys. 74, 1459-1461 (2010).

5. E. G. Ekomasov, R. R. Murtazin, Sh. A. Azamatov. Phys. Solid State. 54, 1584-1590 (2012).

6. V. Nazarov, R. Shafeev. Mod. Phys. Lett. B26, 1250183 (2012).

7. E. G. Ekomasov, R.R. Murtazin, O.B. Bogomazova, A. M. Gumerov. J. Magn. Magn. Mater. 339, 133-137 (2013).

8. I. Morosov, A.S. Sigov. Phys. Solid State. 46(3), 395 (2004).

9. V. V. Kruglyak, A. N. Kuchko, V.I. Finokhin. Phys. Solid State. 46(5), 867 (2004). 
10. O. M. Braun, Yu.S. Kivshar. The Frenkel-Kontorova Model: Concepts, Methods, and Applications. Springer, Berlin. (2004).

11. E.G. Ekomasov, Sh.A. Azamatov, R.R. Murtazin Phys. Met. Metallogr. 105(4), 313 (2008).

12. R.M. Vakhitov, E.R. Gareeva, M.M. Vakhitova, A. R. Yumaguzin. Phys. Solid State. 51(9), 1859 (2009).
13. L. A. Ferreira, B. Piette. Physical Review. E77, 036616 (2008).

14. G. Kalberman. J. Phys. A: Math. Gen. 37, 11603-11612 (2004).

15. E. G. Ekomasov, M.A. Shabalin. The Physics of Metals and Metallography. 101, 1 (2006) S48-S50 p. 